\title{
Overcoming Challenges to Teamwork in Patient-Centered Medical Homes: A Qualitative Study
}

\author{
Ann S. O'Malley, MD, MPH', Rebecca Gourevitch, BA' ${ }^{7}$, Kevin Draper, BA', Amelia Bond, $\mathrm{MPH}^{7}$, \\ and Manasi A. Tirodkar, $P h D^{2}$
}

'Mathematica Policy Research, Washington, DC, USA; ${ }^{2}$ National Committee for Quality Assurance (NCQA), Washington, DC, USA.

BACKGROUND: There is emerging consensus that enhanced inter-professional teamwork is necessary for the effective and efficient delivery of primary care, but there is less practical information specific to primary care available to guide practices on how to better work as teams.

OBJECTIVE: The purpose of this study was to describe how primary care practices have overcome challenges to providing team-based primary care and the implications for care delivery and policy.

APPROACH: Practices for this qualitative study were selected from those recognized as patient-centered medical homes (PCMHs) via the most recent National Committee for Quality Assurance PCMH tool, which included a domain on practice teamwork.

PARTICIPANTS: Sixty-three respondents, ranging from physicians to front-desk staff, were interviewed from May through December of 2013. Practice respondents came from 27 primary care practices ranging in size, type, geography, and population served.

KEY RESULTS: Practices emphasizing teamwork overcame common challenges through the incremental delegation of non-clinical tasks away from physicians. The roles of medical assistants and nurses are expanding to include template-guided information collection from patients prior to the physician office visit as well as many other tasks. The inclusion of staff input in care workflow redesign and the use of data to demonstrate how team care process changes improved patient care were helpful in gaining staff buy-in. Team "huddles" guided by pre-visit planning were reported to assist in role delegation, consistency of information collected from patients, and structured communication among team members. Nurse care managers were found to be important team members in working with patients and their physicians on care plan design and execution. Most practices had not participated in formal teamwork training, but respondents expressed a desire for training for key team members, particularly if they could access it on-site (e.g., via practice coaches or the Internet).

CONCLUSIONS: Participants who adopted new forms of delegation and care processes using teamwork approaches, and who were supported with resources,

Received April 29, 2014

Revised August 12, 2014

Accepted October 4, 2014

Published online November 11, 2014 system support, and data feedback, reported improved provider satisfaction and productivity. There appears to be a need for more on-site teamwork training.

KEY WORDS: Primary health care; Patient care team; Patient-centered medical home; Quality of health care; Practice management.

J Gen Intern Med 30(2):183-92

DOI: $10.1007 / \mathrm{s} 11606-014-3065-9$

(c) Society of General Internal Medicine 2014

\section{INTRODUCTION}

The imbalance between primary care demand and capacity in the U.S. creates barriers to patient access and contributes to physician burnout. Many believe that enhanced interprofessional teamwork can promote more efficient primary care delivery ${ }^{1-4}$ Inter-professional teamwork has been defined as "the provision of comprehensive health services to individuals, families, and/or their communities by at least two health professionals along with patients, family caregivers and community services who work collaboratively on shared goals within and across settings to achieve care that is safe, effective, person-centered, timely, efficient and equitable. "1,5 The primary care team typically includes a lead clinician, such as a physician or nurse practitioner, and other key personnel including nurses, medical assistants (MAs), care managers, practice managers, clerical staff, as well as others, when available (e.g., behavioral health and pharmacists). The utilization of teamwork enables organized care delivery that makes efficient use of patients' and providers' time and frees more highly trained clinicians to focus on complex aspects of patient care. ${ }^{6-8}$

Despite extensive talk about teamwork within patientcentered medical home initiatives and among policymakers, there is little practical information available to primary care providers on how to function as teams. There is a welldeveloped body of literature on teamwork related to industrial, military, and more recently, to hospital settings, ${ }^{9}$ but literature with regard to teamwork in primary care facilities, which differs greatly from that in a hospital setting, is in the early stages. The IOM and others have noted that there is "very little data" regarding effective teamwork in primary care, citing a 
need for examination of the characteristics of highly effective teams and how they are implemented. ${ }^{1,5,10,11}$ Researchers $^{2-}$ $4,12-15$ have begun to examine these topics, but much remains to be explored in terms of how primary care practices develop functional teams.

To help fill the information gap, this study examines how selected primary care practices have developed teams and how they have overcome common challenges to team-based primary care. It also explores implications for care delivery and policy.

\section{METHODS}

\section{Identification of Participants}

To identify practices using an empiric measure of highfunctioning teams, we started with a list of practices recognized as PCMHs as of February 2013 via the NCQA 2011 PCMH tool, ${ }^{16}$ which included a new element on practice teams (included in the appendix) focused on eight team characteristics derived from the literature on teamwork. ${ }^{2,12,13,17,18}$

To obtain a range of practice types for our study, NCQA drew a random sample of PCMHs stratified by geographic region and by variables covering both practice size (number of physicians/independent clinicians) and ownership (e.g., physician-owned vs. hospital- or health system-owned vs. community health center (CHC), etc.). The NCQA divided practices within geographic regions into two groups: (1) those achieving $100 \%$ of the points on the practice team element (high team score) and (2) those obtaining 0-25\% of the points on the practice teams element (lowest team score). Our goal was primarily to interview practices with high scores, but we included one low-scoring practice per region for qualitative comparison. We were not aware of the practice team element scores at the time of the interviews.

For confidentiality reasons, NCQA made the initial outreach to the practices, at which time they described the study goals and assured the practices that participation would have no bearing on their PCMH recognition and that participants would not be identified. NCQA sent a list of practices that consented to an interview to our research group, and our research assistant conducted a screening phone call to confirm practice characteristics and to identify a lead clinician who could best speak to the topic of primary care teamwork at the practice. Then, during the lead clinician interview, a second (and if possible, a third) informant involved in the operational aspects of teamwork at the practice was identified.

Given the project objectives and budget, our goal was to interview between 25 and 30 practices. After completing 63 interviews at 27 practices from across the strata, we were no longer hearing substantively new information (themes were consistently being repeated), and we concluded our outreach.

\section{Interview Protocol}

The interview protocol was partially based on a literature review, and included questions summarized in Table 1. At the beginning of each interview, we defined the term "primary care team" for respondents as "two or more people working together to provide primary care for patients."

\section{In-Depth Interviews}

Interviews occurred between May and December 2013. Every practice completed at least two separate interviews (a lead physician and a second practice team member), and several completed three interviews. Interviews lasted 45-60 minutes and were conducted via telephone by a senior researcher and trained research assistant. The research assistant typed verbatim notes during each interview, which were reviewed by the senior interviewers.

\section{Analysis}

To avoid prematurely imposing a prescribed conceptual framework for teamwork onto the interview data, we conducted a preliminary reading and first-stage analysis that evaluated informant responses using a combination of descriptive and emergent coding. ${ }^{19}$ Three research team members reviewed every interview in its entirety and created separate lists of potential themes and codes

Table 1. Summary of Key Protocol Questions for In-depth Interviews of Practice Respondents

1. Who is on your primary care team?*

2. What are the key challenges to teamwork that you face in caring for patients? *

3. What challenges do you face in quality improvement?*

4. How did your practice overcome each of these challenges to teamwork?*

5. How does your practice identify tasks and delegate roles?*

6. Explain the tasks/roles of each person in the practice.*

7. How did the roles and responsibilities of each team member change as you moved toward more structured team functioning? *

8. Did anyone in your practice take part in formal team training?

9. How do electronic health records (EHRs) facilitate/pose challenges to teamwork? (Findings largely summarized in separate paper)*

10. How does working as part of a team affect continuity of care between the patient and physician?

11. How do you organize your physical space and staffing with respect to the team?

12. Do regulations, or their interpretation, influence how you function as a team?

The full interview protocol is available from the lead author.

* Physicians were asked all of the questions listed. Other respondents were posed the questions followed by an asterisk as well as those questions particularly pertinent to their self-defined roles in the practice. 
from the transcripts. The team revisited the code lists in several meetings and came to agreement on the definitions for each through an iterative process.

To determine the conceptual framework on teamwork that best fit the themes and codes we had identified in this first phase, we used the list of available frameworks from our prior literature review ${ }^{1,2,9,20}$ and settled on the Ghorob and Bodenheimer framework, ${ }^{2,12}$ which identifies five key elements of team building: 1) Defined Goals, such as specific measurable operational objectives; 2) Systems, including the physical arrangement of team members and clinical systems (e.g., standing orders for common situations); 3) Division of Labor, including clear task definition and role assignment; 4) Training of team members on their functions; and 5) Communication, including communication structures (e.g., instant messaging and informal face-to-face gatherings of the clinical team known as "huddles" ${ }^{21,22}$ ) and processes (e.g., feedback, conflict resolution).

After matching codes/themes to each of the five elements (in some cases codes/themes supported more than one element), the second stage of our analysis involved two team members collaboratively applying the codes to the data in Atlas.ti. ${ }^{23}$

\section{Participants}

The 63 in-depth interviews included 60 primary care practice participants in 27 practices from 17 states as well as three national experts on teamwork who were also experienced clinicians. Practices comprised a range of sizes, ownership types, and EHR use. The practice respondents included 22 physicians, 3 nurse practitioners/physician assistants, 7 RNs/ LPNs, seven MAs, 12 administrative/front desk staff, and 9 practice managers. Practice and respondent characteristics are provided in Table 2.

\section{RESULTS}

Primary care teams had certain common characteristics. Several practices with clearly defined roles and practice support used a core clinical team ratio of one lead clinician to two medical assistants to three exam rooms. Larger practices divided themselves into smaller functional team units. If the practice had nurses, these ratios varied. In most practices, MAs were paired with the same clinician on a day-to-day basis.

In addition to a core clinical team, some respondents had full-time or part-time care managers whose roles included ongoing between-visit communication and coordination of care for patients with complex health needs. ${ }^{24} \mathrm{~A}$ few practices, particularly community health centers and those in larger systems, included other team members (e.g., behavioral health workers, pharmacists)
Table 2. Respondent and Practice Characteristics

\begin{tabular}{|c|c|}
\hline & Frequency \# \\
\hline Respondent total & 63 \\
\hline \multicolumn{2}{|l|}{ Respondent type } \\
\hline Physician & 22 \\
\hline Nurse practitioner/physician assistant & 3 \\
\hline Medical assistant & 7 \\
\hline $\mathrm{RN} / \mathrm{LPN}^{*}$ & 7 \\
\hline Practice manager & 9 \\
\hline Administrative \& front desk staff* & 12 \\
\hline National expert on teamwork in primary care & 3 \\
\hline Practice characteristics (total) & 27 \\
\hline \multicolumn{2}{|l|}{ Primary care population served } \\
\hline Adult and pediatric & 17 \\
\hline Pediatric only & 3 \\
\hline Adult only & 7 \\
\hline \multicolumn{2}{|l|}{ Practice type } \\
\hline Independent physician-owned practice & 15 \\
\hline Hospital- or health system-owned ${ }^{* *}$ & 8 \\
\hline $\mathrm{CHC} / \mathrm{FQHC}$ & 2 \\
\hline Independent safety-net clinic & 1 \\
\hline Military & 1 \\
\hline \multicolumn{2}{|l|}{ Practice size (number of physicians) } \\
\hline $1-2$ & 8 \\
\hline $3-10$ & 12 \\
\hline $11-20$ & 3 \\
\hline $21-50$ & 1 \\
\hline$>50$ & 3 \\
\hline $\begin{array}{l}\text { Electronic health record use (many still } \\
\text { partially use paper) }\end{array}$ & 27 \\
\hline \multicolumn{2}{|l|}{ Region } \\
\hline Mid-Atlantic & 4 \\
\hline Midwest & 3 \\
\hline Mountain & 2 \\
\hline New England & 5 \\
\hline Northeast & 5 \\
\hline Plains & 2 \\
\hline Southeast & 4 \\
\hline \multirow{2}{*}{\multicolumn{2}{|c|}{$\begin{array}{l}\text { West } \\
\text { PCMH recognition level (NCQA) }\end{array}$}} \\
\hline & \\
\hline Level 1 & 0 \\
\hline Level 2 & 5 \\
\hline Level 3 & 22 \\
\hline \multicolumn{2}{|l|}{$\begin{array}{l}\text { Performance on practice team element } G \text { in } \\
2011 \text { NCQA PCMH tool }\end{array}$} \\
\hline $0-25 \%$ of points & 7 \\
\hline $100 \%$ of points & 20 \\
\hline
\end{tabular}

*5 respondents served primarily in a care coordinator role (as defined in text).

**Practices included one Kaiser practice working under capitation; otherwise, most practices worked predominantly in fee-for-service arrangements, with some supplementary PCMH payments.

who helped with chronic care management, medication reconciliation, education, and counseling. Almost every respondent described the patient as a team member, noting that efforts to function more effectively were focused on patients. Patient roles were particularly focused on carrying out care plans, self-management support, and personal decision-making.

Table 3 summarizes participants' descriptions of roles and responsibilities and how they changed as teams became more structured. In small to medium-sized practices, where RNs may be too costly, some MAs are increasingly gathering information from patients with the assistance of physician-developed EHR templates and performing routine clinical tasks such as vaccinations and specimen collection (Table 4). Physicians in the four practices that 
Table 3. Primary Care Team Member Roles and Responsibilities Have Changed with Increased Teamwork

How role changed with increased teamwork (illustrative examples)

Primary care physician

Changes:

- More reliance on other team members for paperwork and some aspects of ongoing patient education

- More supervision of team member functioning, greater leadership role

- More thought to how clinical care is broken down into care processes and tasks

- Physician time spent up front creating EHR templates for line of clinical questioning for staff to help gather patient information

- More time available to focus on patients

- Population health management for panel of patients

- Team leader or co-leader (in most practices)

In addition to above changes, also continues to:

- Maintain an ongoing relationship with patient over time

- Provide comprehensive whole-person care, including preventive, acute, chronic, and complex care needs

Nurse practitioner/physician assistant

Changes:

- Some NPs taking lead on care process improvement

In addition to above changes, also continues to:

- Role varies by practice — some have own panel; others focus on same-day visits/walk-ins

Registered nurse $(\mathrm{RN})$ and licensed practical nurse (LPN)

Changes:

- More patient education (e.g., some practices have started RN-led group education classes)

- Some have transitioned toward more patient care (e.g., reinforcing care plan with patient) and less clerical work

- With EHR, nurses do more history-gathering than they did in the past

In addition to above changes, also continues to:

- Perform phone or in-person triage where applicable

- Manage doctors' in-baskets

- Gather hospital discharge summaries and follow up with patient by phone

- Answer patient questions from e-mail/portal

- Manage patient registry

- Coordinate and track referrals

- Medication reconciliation

- Monitor blood work, lab orders, administer vaccines

- Handle prescription refills

Nurse care manager (relatively new role, most often filled by RN)

- Works with complex patients on care plan, goals, education; monitor periodic labs and results

- Uses practice registry to identify care gaps and identify need for population management

- Care transitions: works with hospitalists on patient admissions/discharges and communicates info to PCP

Medical assistant (MA)

Role of MA substantially increased with more structured teams (see separate Table 3)

Front desk staff

Changes:

- Some paired with a specific team unit (e.g., MD/MA/front desk person as a pod)

- More emphasis on improving the patient experience, i.e., greeting \& customer service throughout visit

- More patient outreach, e.g., encourage patient to register on practice's patient portal, if available

- More paperwork formerly done by clinical staff

In addition to above changes, also continues to:

- Obtain demographic information from patients and enter into EHR

- Request prior medical records (new patients)

- Obtain information regarding reason for visit prior to the office visit so that team has this information for their huddle

- Schedule follow-up visit and tests

- Handle insurance information/issues, assist with billing and referral tracking

- Note patient phone calls in EHR and direct calls to appropriate provider

Practice manager

Changes:

- Helps coordinate all-staff meetings

- Go-to person in some practices for non-clinical staff personnel issues related to teamwork

In addition to above changes, also continues to:

- Oversee practice management, aspects of reporting for quality improvement and meaningful use

- Oversee coding and billing 
Table 3. (continued)

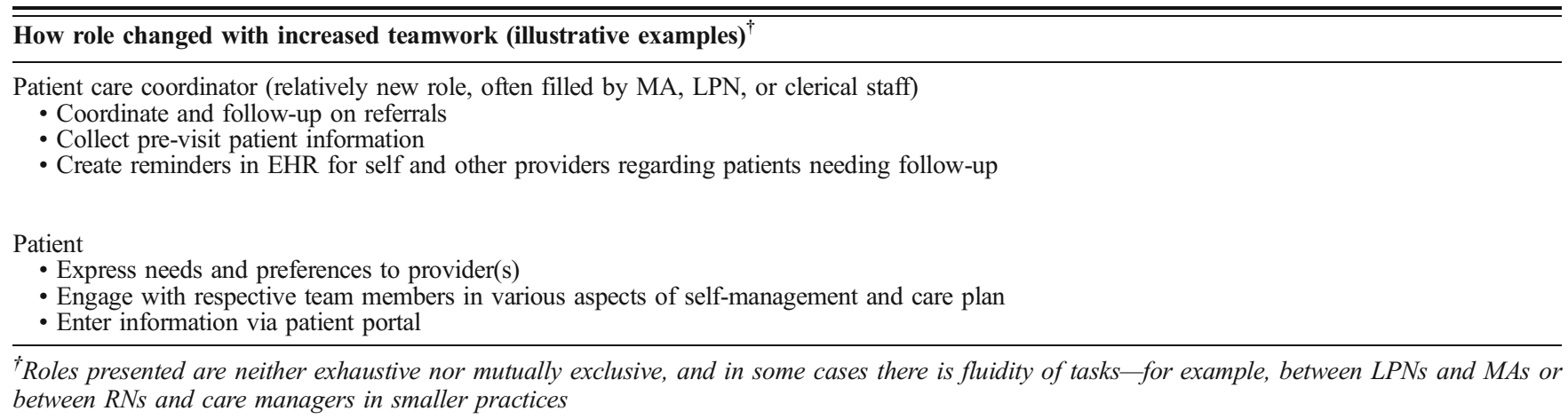

relied most on templates noted that they had to spend time creating templates and protocols to ensure that MA roles were safe for patients and consistent with evidence-based care.

Methods for overcoming common challenges in moving to team-based primary care are presented in Table 5. The following illustrative examples are organized within the five primary care teamwork elements. ${ }^{2,12}$

\section{Goals}

Physician and staff buy-in for the alignment of practice members around common goals in patient care and workflow, as well as staff involvement in developing work flow processes, was aided by data demonstrating the results of team-based care. A Colorado family physician used quality reports generated from the practice's EHR data by an independent practice association (IPA): "Doctors love data, but only when they own it... and when the tools you are using for clinical care as part of a team have improved because of the providers' input, you have them, hook, line and sinker."

Getting provider and patient buy-in for creating and using patient care plans (individualized plans of care that include treatment goals and how the team will work with the patient toward those goals ${ }^{25}$ ) was one of the most significant goalsetting challenges. A physician noted, "It's been hard to get people on the team to understand the purpose behind the care plan," and that a "lack of staff infrastructure" posed implementation challenges. Similarly, participants stated that patients often did not understand the purpose of the care plan and did not comply with it.

Scores on NCQA team element items indicate that lowscoring practices did less well with training about selfmanagement support (which typically includes care plans). None of the low-scoring practices raised the topic of care plans during their interviews, while respondents from highscoring practices were more likely to mention care plan challenges (suggesting a greater awareness of the care plan concept). The one solution noted for care plan implementation challenges involved nurse care managers engaging patients and their physicians in designing, revisiting as needed, and executing the plan.

\section{Division of Labor}

To overcome the challenge of physician reluctance to delegate, the most common strategy was to introduce task delegation incrementally, starting with items that physicians could agree were safe to hand off to MAs (e.g., standing orders for urinalysis for UTI symptoms and pneumococcal vaccination). Next, some physicians developed templates within their EHR to guide staff when gathering information from patients with common complaints. Third, having outside practice coaches or facilitators teach teamwork and verify nurse competency reassured physicians about delegating tasks. As MA/RN interaction with patients increased, some physicians gained comfort by talking with patients about exactly what messages they had received.

To address the commonly noted challenges of role definition, many practices used part of their all-staff meetings to allow staff members to describe their daily tasks so that others could understand the respective roles and identify where tasks could be streamlined. Team training and practice coaches were helpful in this area.

Offloading of routine tasks to MAs and LPNs resulted in increased job satisfaction for physicians in several practices, who could instead focus on patients' more complex and personal needs. MAs reported improved job satisfaction from feeling more involved in patient care and becoming more than "just the vital signs person."

\section{Communication}

Maintaining real-time and structured communication was a common challenge, particularly for practices with low scores on the NCQA practice team element. Of the 27 practices in this study, 23 mentioned huddles as key to maintaining structured communication within their teams. A physician leader said that "regular huddles are one of the first things all practices should 
Table 4. Optimizing the Role of Medical Assistants on the Primary Care Team

Medical assistant tasks*—examples noted by practice respondents)

Collect information prior to patient visit (usually in the week before visit): Compile prior test results/consultation reports, reason for appointment, etc. Identify when patients are due for tests/gaps in care, using guidelinebased protocols

Order or queue up for the physician (depending on state laws) necessary tests based on standing orders:

Eye exam

Mammograms (routine screening based on guideline eligibility)

Colorectal cancer screening (routine screening based on guidelines) Identify patients needing vaccinations (administer vaccination, depending on state regulations)

Collect information with patient in office:

Log the chief complaint into the record, which physician will verify/ edit as needed

Ask screening/standard questions of patients working off of template, which is built into EHR in some practices

Update info in EHR; e.g., verification of medications

Perform tasks day of visit:

Rooming patients

Take vital signs (adults) including height/weight and head circumference (children)

Collect samples within their scope: e.g., blood draws, urinalysis, when indicated based on protocol

Administer vaccinations (if allowed by state)

$\mathrm{EKG}$, if indicated, based on physician-created algorithm for certain patients (e.g., preop visit)

Depression screening

Chronic disease monitoring and patient education (upon physician request) based on protocols/training by practice; e.g., counseling on tobacco cessation, diabetes, asthma, hypertension

Reinforce goals \& enlist patient engagement in chronic disease management after physician encounter

For patients requiring additional help, escort them to front desk after physician visit to schedule needed services

Handle incoming patient requests:

Manage messages that come through patient portal and send to appropriate team member

Handle patient phone calls regarding matters that do not require clinical triage

Cross-training as receptionist/scheduling

Ongoing tasks (not visit-specific):

Monitor lab results as they come into EHR from labs and ensure that they go to appropriate team provider

Provide patients with normal lab results

Sort/screen messages in EHR in-basket (when given access by provider)

Referral tracking: assist patients in arranging appointment with specialist upon PCP referral, track referrals

Scan outside consult reports [that] are paper as they come in and send them to the physician's approval queue inbox so that it comes up as a task for the physician to view

Run quality reports from registry based on a protocol

"Queue up" the less clinical data from EHR for ACO \& MU reporting requirements - e.g., social history, BMI, demographics - using a checklist/protocol as guidance

Provide input on how MAs can best manage the tasks they are assigned - e.g., what computer hardware works best for them (desktop vs. tablets) - and provide that feedback to practice leader

*In some practices, nurses perform some of these roles (see Table 3).

do." Learning to huddle typically entailed heavy involvement of a leader (physician or PCMH champion) and observation of huddling by other teams. In some practices, getting huddles "down to a science" took over a year.

Huddles generally were used to clarify the "game plan" of what the team would be doing for patients scheduled to come to the office that day. They typically took place at the beginning of morning or afternoon office hours and lasted 5 to 10 minutes (but allowing teams flexibility on when they huddled was helpful), and usually included the physician and MAs or the physician and MA and nurse. In some cases, a front-desk team member was part of the huddle, but usually the MA or nurse relayed information relevant to front-desk staff after the huddle. They often began by taking stock of which staff members were present in the office, followed by a quick discussion of each scheduled patient, the special issues or tasks for particular patients, and, when such tasks were not already part of predetermined team member roles, identification of who would carry them out.

Some practices used a paper or electronic "huddle sheet", created with a customized EMR template and populated with data from the EHR and from the previsit planning work that the MA or nurse had completed (typically) in the two weeks prior to the scheduled visit. In practices where part-time or shared staff (e.g., nutritionists, nurse care managers, and behavioral health specialists) were not always on-site, a standardized process for notifying them of relevant information from the huddle would be accomplished via the EHR.

Several other techniques also helped to minimize communication breakdowns. Chief among these was colocation of the MD/RN/MA in order to enable in-person communication. Some respondents mentioned creating a "safe" culture" in which people could feel comfortable providing feedback without threat of reprisal, and in some instances this was facilitated by creating a "go-to" person for staff to express concerns or ask questions. Use of the EHR, including instant messaging features and task assignment into different "buckets" or "task lists" for team members, also facilitated communication.

Feedback from many respondents, as well as a wide body of literature, suggests that continuity of care between clinicians and patients is a prerequisite to trust and effective communication. ${ }^{26}$ Physician participants generally felt that teamwork enhanced interpersonal continuity with patients, as the necessary data to inform a patient encounter had been collected before the visit, freeing them to spend more of the visit discussing issues most important to the patient. To maximize patients' familiarity with team members, most practices restricted the core clinical team to two or three people who had ongoing contact with the patient, and some handed patients a card with the names, titles and roles, and contact information of the key team members. 
Table 5. How Practices Overcame Challenges to Teamwork

\begin{tabular}{l}
\hline Challenge \\
Goals \\
Gaining provider and staff buy-in to teamwork \\
Need for effective leadership \\
Engaging patients in goal identification and \\
achievement
\end{tabular}
achievement

\section{Division of labor}

Physician reluctance to delegate

Poor role definition

Staff retention

Communication

Communication breakdown

Initiating huddles \& getting providers to attend

"Difficult personalities"

Risk of disrupting interpersonal continuity of care with patients as team grows

Systems

Sliding back to pre-team behaviors

Protect physicians from non-clinical tasks

Limited resources for hiring

Fee-for-service emphasizes visit volume

Part-time staff, transitional staff, and different shifts
Solutions practices used to overcome challenges

"Once our priorities are clear, the plan for what we do follows." (Physician)

- Present data on results of changes in care processes to demonstrate merits

- Encourage a culture where staff support one another

- Engage the staff to identify best practices and optimal workflow

- Leaders need to recognize \& acknowledge problems in institution's culture and to engage staff to address them

- Recruit skilled leaders

- Formal leadership training

- Care manager or physician outreach to patient to come for visit so all three can sit together to reinforce goals and discuss care plan

- Encourage patient to use electronic portal to enter data (e.g. weight, blood pressures, glucose levels) and to access educational materials

"We can't just go directly to the MAs and tell them what their new responsibilities are without involving them in that decision." (Physician leader)

- Introduce delegation of tasks by provider to MA incrementally

- Create electronic templates to help ensure appropriate information collection by MA

- Outside team verifies competency of nurses to handle newly delegated tasks

- Physicians more willing to delegate when team staffing is stable; e.g., informal agreements that MAs would stay at practice if they receive additional training

- Pre-visit planning sheets to maximize visit efficiency \& avoid missing issues

- In an all-staff meeting, each person walks through their daily tasks to help one another understand respective roles \& to identify areas for process improvement

- Delegation of tasks to various staffs using task lists or "buckets"

- Fair distribution of tasks, including the more mundane tasks

- Higher pay may be needed to retain effective and productive RNs, LPNs, or MAs

"In an average practice, there are often lines of authority rather than open communication." (Primary care physician)

- "Having common goals facilitates structured communication"

- Organize office space to co-locate MD/RN/MA for ease of live communication

- Daily huddles to reinforce responsibilities and discuss patient-specific issues

- Address communication problems in meetings

- EHR as tool to help assign tasks to team members; e.g., electronic to-do lists or task lists (especially useful for part-time, off-site staff, e.g., nutritionists, care managers)

- Create a "safe" environment for team members to ask questions

- Nurse or practice manager as go-to person for communication gaps within team

- Cross-pod and cross-site collaboration to share lessons

- White board in hallway near nurses desk to indicate pending visit tasks

- Physicians/staff learn huddle process by observing others huddle

- Allow teams flexibility on when they huddle (AM vs. PM vs. both)

- Use populated pre-visit planning form to guide huddle

- Align team personalities \& shifts that work well together

- Teach providers to communicate in a nonjudgmental manner

- If a person isn't functioning well in a given role, try to repurpose that role

- Evaluation, disciplinary action, and sometimes termination may be necessary

- When hiring, emphasize that the person will be expected to act as team member

- With pre-visit data collection, most physicians felt that teamwork freed them to spend more of the visit discussing issues most important to the patient

- Team hands patient a card with their team members' names, titles, \& roles

- Pair same RN/MD or MA/MD in teams to allow them to learn one another's styles and to ensure they are the key people interacting with the patient

- Schedule patients with their PCP unless unavailable

"All the providers were just kind of sucking up the work themselves. They weren't bouncing phone encounters back to us." (Practice manager describing why they automated e-mail) phone appointment requests to go directly to front desk rather than to physician)

- Build checks into system (e.g., practice manager tracks task completion in EHR)

- Weekly staff meetings \& daily huddles to address problems in a timely fashion

- Some practices assigned a front desk person per pod/teamlet

- Establish rules and criteria for scheduling provider time

- Care managers focus on most at-risk patients

- Most practices used their PCMH funding to hire a part-time care manager or RN

- Large system switched to model where doctor was accountable for own expenses, including

MA salaries, to allow allocation of funds to structure their team

- Try to maintain consistency in leadership role

- Maintain continuity between the longstanding staff (PCPs, nurses) and patients

- Require team huddles to ensure rotating staff are on same page

- Cross-train staff on tasks within their scope/skill level 
Table 5. (continued)

\begin{tabular}{|c|c|}
\hline Challenge & Solutions practices used to overcome challenges \\
\hline Incorporating "shared" cross-practice staff & $\begin{array}{l}\text { - Co-locate staff (e.g., shared care managers) when possible } \\
\text { - If care manager can't attend huddles, share relevant notes from huddles with them } \\
\text { - If possible, replace the outside care coordinator with someone from within the practice }\end{array}$ \\
\hline Volume of performance measures required & $\begin{array}{l}\text { - Divide tasks, for example: 1) Front desk staff flags patients with diabetes or tobacco use on the } \\
\text { schedule; 2) MA audits those charts, completes pre-visit planning forms, \& gives to team; 3) } \\
\text { clinicians do the tasks that MA cannot do } \\
\text { - Larger entities (IPA) can help practices with reporting, data aggregation }\end{array}$ \\
\hline Training & "The toughest thing is to avoid going back to old habits once training is done" \\
\hline Finding time and resources & $\begin{array}{l}\text { - Most common training was from within the practice by physician leaders } \\
\text { - Key team members need to participate in training, not just the lead physician } \\
\text { - Staff attend "super-user training to get clearance to create EHR templates." } \\
\text { - Some practices have been trained in teamwork by their larger system } \\
\text { - Use of practice coaches, e.g., HealthTeamWorks, CO (www.healthteamworks.org) }\end{array}$ \\
\hline
\end{tabular}

\section{Systems}

Systems-based challenges, both within and external to the practice, were the themes most frequently mentioned by respondents, and typically required system-based solutions. For example, a common problem (particularly among practices with low practice team element scores) was the tendency to "slide back toward pre-teamwork behaviors." To address this, many high-scoring practices used a combination of daily huddles and checks built into their electronic system (tracking of task completion in the EHR) to ensure that problems were quickly corrected.

One example of the way that systems can be used to support teamwork involved diabetes management and the volume of related performance reporting in the face of "only 15-minute visits." In response, a few practices instituted a diabetes clinic day for each provider. As a nurse in such a practice noted, "If a patient's A1c is greater than 9, they are given a 30-minute appointment." In these instances, the health educator and care coordinator are present, and the MA who works at the diabetes clinic also has more extensive training in diabetes. The nurse noted, "The providers and patients are happy, and we have some examples of patients lowering their A1c."

External system challenges to teamwork included insufficient staff and current fee-for-service incentives that emphasize office visits over population management and care coordination. To partially address these challenges, a Maryland practice negotiated with the larger health system to which it belonged to switch to a compensation model in which physicians were made responsible for their own MA salaries, enabling them to allocate funds to structure their teams for maximum efficiency and productivity.

Some practice respondents and one national expert noted fear of "running afoul of regulations" as hampering role delegation. A provider echoed the sentiments of clinicians in two large organizations when she noted that their ability to use standing orders was limited because their compliance department feared that the EHR could give the appearance that the
MA was ordering the service. An $\mathrm{RN}$ in a California practice overcame this issue by requiring the provider to authorize the standing orders in advance of patient visits.

\section{Training}

Respondents mentioned challenges to training such as "lack of time to attend training" and the lack of awareness of training resources. Most practices had not undergone formal teamwork training. Some had a physician leader who had read about teamwork and then championed change within the practice. In Colorado, where HealthTeamWorks ${ }^{27}$ is active, practices used practice coaches ${ }^{28}$ and the military practice used PC TeamSTEPPS. ${ }^{20}$ Other practices, however, could not identify formal teamwork training. Many had participated in PCMH learning collaboratives and/or received some guidance from their larger parent organizations or IPAs, but commented that these often lacked teamwork training. Respondents from three practices also noted a need for more formal team training around patient selfmanagement and care plans.

\section{DISCUSSION}

This paper provides practical details on how practices have developed solutions to address common challenges to teamwork. It includes new approaches as well as confirmation of previously described tools. ${ }^{3,4,9,12,24,27-32}$ These included using data to show the team how patient care improves with teamwork, incremental delegation by physicians, ${ }^{3,4}$ engaging staff in workflow redesign, creating a safe culture for feedback and questions, ${ }^{9}$ using outside coaches or practice facilitators, ${ }^{27,28}$ huddles, ${ }^{21,22}$ using EHR templates to guide data collection by MAs and nurses, and tracking task completion to help prevent sliding back to pre-teamwork behaviors. 
When task redistribution maintained patient care safety, job satisfaction and interpersonal continuity of care improved both for physicians and for MAs and nurses who appreciated greater involvement in patient education and decisions about team care processes. Respondents generally believed that teamwork helped to avoid neglecting important patient care issues.

External system challenges (e.g., fee-for-service payment, resources, regulations) $)^{3,33}$ were most problematic because they were out of respondents' control. Others have suggested that when teams lack formal authority to alter such external systems, they are particularly reliant on effective clinical leadership, ${ }^{34}$ so offering training to potential leaders in how to change care processes may help practices enhance performance and meet patients' needs in a manner consistent with scientific evidence until such external challenges are resolved.

Limitations. We cannot generalize from this study to all primary care practices. The practice team element of the 2011 NCQA PCMH tool has not yet been widely validated, but its key elements are based on teamwork literature and feedback from providers, and it is the only practice-based sampling frame at present that provides some empiric indicator of teamwork functioning in primary care practices on a national scale.

This study's findings have implications for practices working toward PCMH recognition as well as those already recognized as PCMHs, in addition to those simply interested in enhancing their teamwork. The provision of venues and resources for practice teams to learn from one another across practice sites, both in person and online via Web-based learning, has the potential to improve teamwork by allowing practices to share lessons learned and to model behaviors/roles for one another. Areas particularly ripe for such shared learning include effective role delegation, structured communication strategies (e.g., huddles), ways to avoid sliding back into pre-teamwork behaviors, and team roles for efficient population management. Such shared learning, particularly if facilitated by persons knowledgeable about on-the-ground use of EHRs in primary care, has the potential to help practices in improving the quality of their teamwork.

From a policy perspective, practices expressed a need for more staffing resources to enhance teamwork in care plans and patient self-management, funding for practice coaches, and assistance in better understanding the implications of regulations (e.g., scope of practice) with respect to teamwork.

As recognized PCMHs, the practices we included likely have better resources and may be more highly motivated than typical practices in the U.S., but their awareness and use of resources or curricula to support primary care teamwork was scant. We hope that the present study, in addition to current work in the field, ${ }^{35}$ will add to the existing body of research in helping practices devise ways to function as more effective teams. From participants' comments, it is clear that the demands of primary care make it challenging for staff to leave the practice for lengthy periods of time to engage in formal classes, so teaching and tools that can be brought to the practice, or accessed as needed from within the practice, may be most feasible.

Acknowledgments: We are grateful to the Commonwealth Fund for financial support for this study. We also thank the numerous practice respondents who participated in in-depth interviews. We thank Nicole Acciavatti of NCQA for her assistance in the initial outreach to practices.

We also thank Lawrence Casalino, MD, and Richard Ricciardi, NP, $\mathrm{PhD}$, for their feedback on the interview protocol for this study, and Christine Sinsky, MD, for feedback on an earlier version of this paper.

Conflict of Interest: The each authors declare that they have no conflict of interest.

Corresponding Author: Ann S. O'Malley, MD, MPH; Mathematica Policy Research, 1100 1st St., NE, 12th Floor, Washington, DC 200024221, USA (e-mail: aomalley@mathematica-mpr.com).

\section{REFERENCES}

1. Mitchell P, Wynia M, Golden R, McNellis B, Okun S, Webb CE, Rohrbach V, Von Kohorn I. Core Principles \& Values of Effective TeamBased Health Care. Discussion Paper. Washington: Institute of Medicine; 2012. www.iom.edu/tbc.

2. Ghorob A, Bodenheimer T. Sharing the care to improve access to primary care. N Engl J Med. 2012;366(21):1955-1957.

3. Sinsky CA, Willard-Grace R, Schutzbank AM, Sinsky TA, Margolius D, Bodenheimer T. In search of joy in practice: a report of 23 highfunctioning primary care practices. Ann Fam Med. 2013;11(3):272-278.

4. Casalino L, Gillies RR, Shortell SM, Schmittdiel JA, Bodenheimer T, Robinson JC, Rundall T, Oswald N, Schauffler H, Wang MC. External incentives, information technology, and organized processes to improve health care quality for patients with chronic diseases. JAMA. 2003;289(4):434-441.

5. Naylor MD, Coburn KD, Kurtzman ET, et al. Inter-professional TeamBased Primary Care for Chronically Ill Adults: State of the Science. Unpublished white paper presented at the ABIM Foundation meeting to Advance Team-Based Care for the Chronically Ill in Ambulatory Settings. Philadephia, PA: March 24-25, 2010.

6. Coleman K, Phillips K. Providing Underserved Patients with Medical Homes: Assessing the Readiness of Safety-Net Health Centers. Commonwealth Fund pub.1395, vol. 85.

7. Mickan SM. Evaluating the effectiveness of health care teams. Aust Health Rev. 2005;29(2):211-217.

8. Shipman SA, Sinsky CA. Expanding primary care capacity by reducing waste and improving the efficiency of care. Health Aff (Millwood). 2013;32(11):1990-1997.

9. Salas E, Frush K. Improving Patient Safety Through Teamwork and Team Training. 1st ed. Oxford: Oxford University Press; 2013.

10. Patel MS, Arron MJ, Sinsky TA, Green EH, Baker DW, Bowen JL, Day S. Estimating the staffing infrastructure for a patient-centered medical home. Am J Manage Care. 2013;19(6):509-516.

11. Shojania KG, Ranji SR, MCDonald KM, Grimshaw JM, Sundaram V, Rushakoff RJ, Owens DK. Effects of quality improvement strategies for type 2 diabetes on glycemic control: a meta-regression analysis. JAMA. 2006;296(4):427-440.

12. Bodenheimer T. 2007 CHCF Lessons Learned when listing the 5 key elements of primary care team building.

13. Grumbach K, Bainbridge $\mathbf{E}$, Bodenheimer T. Facilitating improvement in primary care: the promise of practice coaching. Issue Brief (Commonw Fund). 2012;15:1-14. 
14. Roblin DW, Vogt TM, Fireman B. Primary health care teams: opportunities and challenges in evaluation of service delivery. Innov J Ambul Care Manag. 2003;26(1):22-35.

15. Chesluk BJ, Holmboe ES. How teams work-or don't-in primary care: a field study on internal medicine practices. Health Aff. 2010;29(5).

16. National Committee for Quality Assurance, Patient Centered Medical Home Recognition Tool. PCMH 2011 Standards, Feb.1, 2011, http:// www.ncqa.org/Programs/Recognition.aspx.

17. Scholle SH, et al. Support and strategies for change among small patientcentered medical home practices. Ann Fam Med. 2013;11(supp 1):S6-S13. Funded by AHRQ.

18. Poulton BC, West M. The determinants of effectiveness in primary health care teams. J Interprofessional Care. 1999;13(1):7-18.

19. Miles MB, Huberman MA. Qualitative Data Analysis: An Expanded Sourcebook. 2nd ed. Thousand Oaks: SAGE Publications Inc.; 1994.

20. AHRQ TeamSTEPPS Primary Care Version. Director, Richard Ricciardi. http://www.ahrq.gov/professionals/education/curriculum-tools / teamstepps/primarycare.

21. Institute for Healthcare Improvement. http://www.ihi.org/resources/ Pages/Tools/Huddles.aspx.

22. Fogarty CT, Schultz S. Team huddles: the role of the primary care educator. Clin Teach. 2010;7(3):157-160. doi:10.1111/j.1743 498X.2010.00369.x. Review.

23. Atlas.ti, Version: 5.7.1, Qualitative Data Analysis Scientific Software. (C) 1993-2012 by ATLAS.ti GmbH, Berlin.

24. McDonald KM, Sundaram V, Bravata DM, et al. Closing the Quality Gap: A Critical Analysis of Quality Improvement Strategies (Vol. 7: Care Coordination). Technical Reviews, No. 9.7. Rockville: Agency for Healthcare Research and Quality (US); 2007.

25. Institute for Healthcare Improvement. http://www.ihi.org/resources/ Pages/Tools/MySharedCarePlan.aspx.

26. Rodriguez HP, Rogers WH, Marshall RE, Safran DG. The effects of primary care physician visit continuity on patients' experiences with care. J Gen Intern Med. 2007;22(6):787-793.

27. HealthTeamWorks, CO (www.healthteamworks.org).

28. Taylor EF, Machta RM, Meyers DS, Genevro J, Peikes DN. Enhancing the primary care team to provide redesigned care: the roles of practice facilitators and care managers. Ann Fam Med. 2013;11(1):80-83.

29. Nutting PA, Crabtree BF, Stewart EE, Stange KC, Jaén CR. Journey to the patient-centered medical home: a qualitative analysis of the experiences of practices in the National Demonstration Project. Ann Fam Med. 2010;8(Suppl 1):S45-S56.
30. Anderson PB. Liberating the Family Physician: The Handbook of Team Care for 21st Century Family Medicine. New-port News. Va: Riverside Health System; 2005. http://www.familyteamcare.org.

31. Bitton A, Schwartz GR, Stewart EE, Henderson DE, Keohane CA, Bates DW, Schiff GD. Off the hamster wheel? Qualitative evaluation of a payment-linked patient-centered medical home (PCMH) pilot. Milbank $\mathrm{Q}$. 2012;90(3):484-515.

32. Stewart EE, Stange KC, Jaén CR. Journey to the patient-centered medical home: a qualitative analysis of the experiences of practices in the National Demonstration Project. Ann Fam Med. 2010;8(Suppl 1):S45-S56.

33. Abrams MK, Nuzum R, Mika S, Lawlor G. Realizing Health Reform's Potential. How the Affordable Care Act Will Strengthen Primary Care and Benefit Patients, Providers and Payers. January 2011. http:// www.commonwealthfund.org/Publications/Issue-Briefs/2011/Jan/ Strengthen-Primary-Care.aspx.

34. Bohmer RM. Leading clinicians and clinicians leading. N Engl J Med. 2013;368(16): 1468-1470.

35. Wagner E. http://www.rwjf.org/en/blogs/human-capital-blog/2012/03/ new-rwjf-program-will-study-how-to-use-primary-care-workforce-moreeffectively.html.

\section{APPENDIX}

NCQA PCMH 2011 element 1G: the practice team

The practice uses a team to provide a range of patient care services by:

1. Defining roles for clinical and non-clinical team members

2. Having regular team meetings or a structured communication process

3. Using standing orders for services

4. Training and assigning care teams to coordinate care for individual patients

5. Training and assigning care teams to support patients and families in self-management, self-efficacy, and behavior change

6. Training and assigning care teams for patient population management

7. Training and designation of care team members with regard to communication skills

8. Involving care team staff in the practice's performance evaluation and quality improvement activities 